\title{
Explosionsfeste Transportbehälter - mehr Sicherheit für das Transportwesen
}

\author{
DIPL.-KFF. JOHANNA HAAG \\ FACHGEBIET LOGISTIK, UNIVERSITÄT DORTMUND
}

\section{Zusammenfassung}

Weltweit ist eine Zunahme terroristischer Aktivitäten zu verzeichnen, so dass allgemein damit gerechnet werden muss, dass auch das zivile Verkehrs- und Transportwesen ein bevorzugtes Ziel terroristischer Anschläge darstellt. Mehrfach wurden schon Sprengkörper in Transportmitteln des öffentlichen und zivilen Personen- aber auch Güterverkehrs eingeschleust, um die Bevölkerung durch materielle Zerstörung und massive Personenschäden einzuschüchtern und zu beängstigen. Daher ist eine Anpassung der derzeit im Einsatz befindlichen Transportbehälter an die geänderten Rahmenbedingungen unerlässlich, um auch den Schutz vor Sprengkörpern, die gemeinsam mit dem Handgepäck in Luft-, Land- und Wasserfahrzeuge eingeschleust werden, zu gewährleisten. [Haag2006]

\begin{abstract}
The worldwide increase of terrorist activities leads to the assumption that the civilian transportation and traffic system, too, is a preferred target of terrorist attacks. Several times, explosive charges have already been smuggled into means of public transport and also of goods traffic to intimidate and frighten the population through material destruction and massive personal damage. This is why it is necessary to adapt the containers currently used to the changed conditions, to guarantee the protection against explosives that are smuggled into aircrafts, surface vehicles and watercraft with the hand luggage.
\end{abstract}

Im Rahmen des von dem Forschungskuratorium Textil e. V., Eschborn geförderten und mit finanzieller Unterstützung des Bundesministeriums für Wirtschaft und Arbeit (BMWA) über die Arbeitsgemeinschaft industrieller Forschungsvereinigungen „Otto von Guericke“ e.V. (AiF) durchgeführten ZutechForschungsprojekts Nr. 142 ZBG „Entwicklung von explosionsfesten, textilen Transportbehältern für den Stückguttransport“ untersuchte das Fachgebiet Logistik (FLog) der Universität Dortmund in Zusammenarbeit mit dem Forschungsverbund des Sächsischen Textilforschungsinstituts e. V. in Chemnitz, des FraunhoferInstituts für Kurzzeitdynamik in Freiburg und des Instituts der Feuerwehr in Sachsen-Anhalt die Potenziale von hochfesten, textilen Flächenmaterialien und Nahtverbindungen zur Entwicklung eines explosionsfesten, textilen Transportbehälters. Unterstützt wurden die Forschungsstellen durch ein Kompetenzteam aus Praktikern aller betroffenen Bereiche der Textilbranche, welches das Projekt permanent begleitet und unterstützt hat.

Um eine möglichst hohe Öffentlichkeitswirksamkeit zu erzielen, können Sprengsätze von Terroristen z. B. in Koffern, Taschen, Rucksäcken in Transportmittel des öffentlichen Personen- und Güterverkehrs eingeschleust werden. Diese Güter werden vielfach anschließend in unterschiedlichen Ladungsträgern, z. B. Transportbehältern, zu Transporteinheiten zusammengefasst und in den Transportmitteln verstaut. Bei einer Explosion wird die zerstörende Wirkung des Sprengsatzes durch die Splitterwirkung, die Druckwelle bzw. den schnellen Druckanstieg im Frachtraum, die große Geschwindigkeit der durch die Explosion beschleunigten Gepäckstücke und die hohe Temperatur der Explosionsgase erzeugt. Durch das präventive Verpacken der Stückgüter und Gepäckstücke in spezielle explosionsfeste Behältnisse, kann ein wirksamer Schutz durch das Abfangen der Wirkung des Sprengsatzes erreicht und damit die Zerstörung von Transportmitteln und Gefährdung von Leben verhindert werden. Allerdings können mit den derzeit verfügbaren hartballistischen explosionsfesten Transportbehältern - z. B. HULD (kann man die Abkürzung auflösen) im Luftverkehr - die vielschichtigen logistischen Anforderungen in Luft-, Schienen-, Straßen- und Wasserfahrzeugen nur unzureichend erfüllt werden, da die derzeitigen Einheits-Behältersysteme allein auf die Anforderungen der Luftfracht in Großraumflugzeugen und deren Zusatzsysteme zugeschnitten sind. 


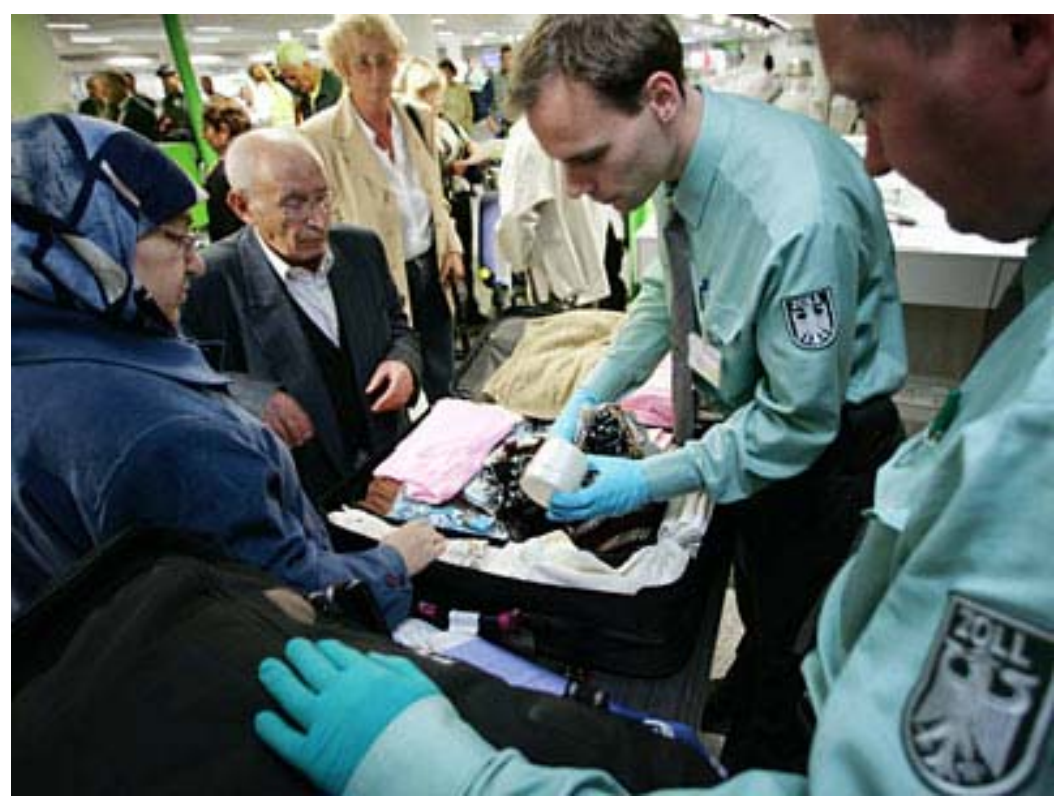

Abbildung 1: Manuelle Prüfung von Gepäckinhalten am Flughafen [Quelle des Fotos: Westdeutscher Rundfunk WDR]

Die Entwicklung von flexiblen, explosionsfesten Transportbehältnissen aus textilen Materialien, wie sie bereits bei ballistischen Funktionssystemen (z. B. schusssichere Westen) werden, kann somit als Alternative mit hoher Anwendungsvariabilität angesehen werden. Der zerstörenden Wirkung eines explodierenden Sprengsatzes kann somit komplex entgegengewirkt, während gleichzeitig die Funktionen von Verpackung und Transportbehältern in Transportmitteln erfüllt werden (Den Satz finde ich ein wenig holprig). Im Projekt wurden deshalb Transportbehälter aus textilen Flächenmaterialien entwickelt, um aus diesen in nachfolgenden Arbeiten durch die Transport- und Verpackungsmittelindustrie universell einsetzbare textile explosionsfeste Transportbehälter fertigen zu können. Diese weichballistischen Transportbehälter sind neuartig und stellen neben den bereits entwickelten hartballistischen Transportbehältern HULD eine weitere Alternative mit breiterer Anwendbarkeit und gleichen Schutzanforderungen dar. Die logistische Innovation besteht darin, dass solche explosionsfesten flexiblen Transportbehälter universell in Verkehrsmitteln unterschiedlichster Art einsetzbar und an die Geometrie des Frachtraumes und ggf. entsprechende Zusatzeinrichtungen anpassbar sind, die keine spezifischen Zusatzvorrichtungen erfordern.

In einem ersten Schritt wurden Untersuchungen von unterschiedlichen flexiblen Transportbehältern, die bereits in der Praxis eingesetzt werden, hinsichtlich ihrer geeigneten und zweckmäßigen Anwendungen in den verschiedensten Straßen-, Schienen- und Wasser- und Luftfahrzeugen durchgeführt und bezüglich ihrer logistischen Funktionen bewertet und abschließend in einem Anforderungskatalog zusammengefasst [Haag]. Dieses logistische Anforderungsprofil wurde um die ballistischen und die brandschutztechnischen Kriterien ergänzt, um Rückschlüsse auf die Entwicklung neuartiger textiler Materialien, die zur Herstellung explosionssicherer Behältersysteme dienen sollen, ziehen zu können. Ausgehend von der Beanspruchung der Behälter zum Gepäcktransport im Flugverkehr erfolgte die Beurteilung zu erwartender Belastungen durch das Einschleusen von vorwiegend synthetischen Sprengsätzen, die sich in beliebige Formen pressen lassen. Aus umfassenden Betrachtungen hierzu konnten Designkriterien formuliert werden, welche für die formalen Gestaltungsmerkmale von Transportbehältern von Bedeutung sind. Die Gestaltung der textilen Strukturen war dabei so auszuführen, dass die im Explosionsfall auftretenden Belastungen flächig verteilt werden und eine möglichst hohe Energievernichtung stattfindet.

Aufgrund der zuvor beschriebenen Anforderungen wurden verschiedene textile Flächenmaterialien unter Verwendung von Hochleistungsfasern als Muster hergestellt und in drei unabhängigen Testserien hinsichtlich der Einhaltung der ballistischen und brandschutztechnischen Vorgaben geprüft.

Zunächst wurden die unterschiedlichen Materialien in einer repräsentativen Nachstellung einer Explosion bezüglich Flammendurchschlag, Brandhemmung, Nachbrennverhalten und Wärmeleitung untersucht und Hochtemperaturen ausgesetzt (vgl. Abb. 2a - 2c). Hierbei wurden die Schadensverläufe jeweils detailliert untersucht und die Schadensbilder, welche sich infolge einer vorgegebenen Einwirkdauer ergaben, analysiert und miteinander verglichen. 


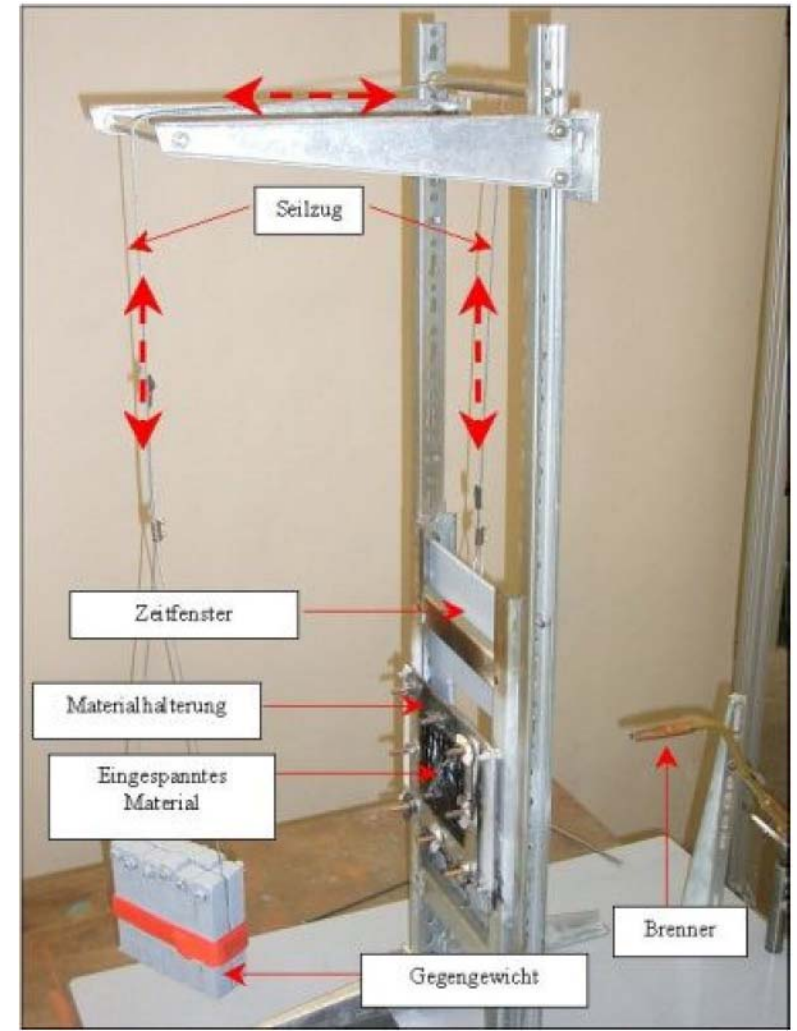

Abbildung 2a: Apparatur „Hochtemperaturtest (HHT)“

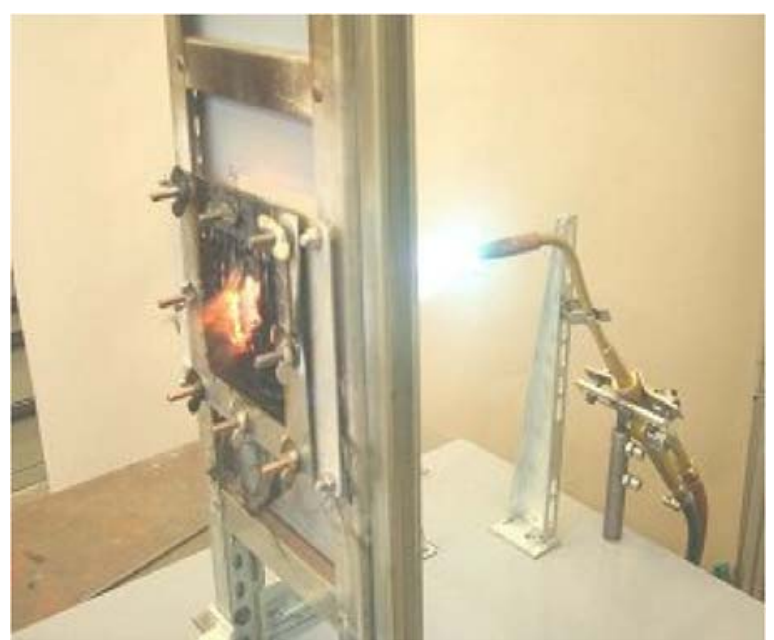

Abbildung 2b: HHT im Betrieb

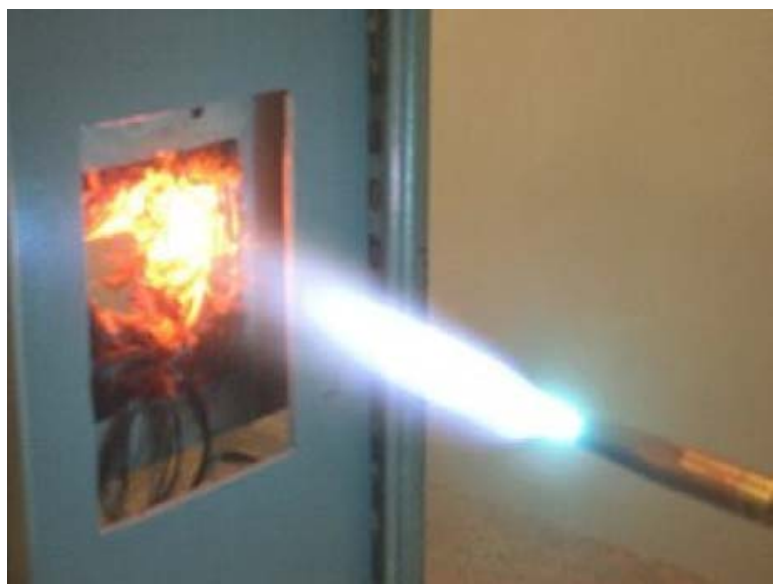

Abbildung 2c: Beflammung des Materials

Im nächsten Schritt sind die gleichen Materialien mit Einsatz eines Druckrohres zum Bersten, sprich dem plötzlichem, schlagartigem Versagen des Materials bei Druck, gebracht worden. Durch ein Druckrohr kann der bei einer Explosion wirksame Gasdruck in einem Behälter, der durch Explosion von innen beansprucht wird, simuliert werden. In der Versuchsserie konnten die spezifischen Drücke bestimmt werden, bei denen ein Versagen der textilen Materials erreicht wird. Abschließend wurde in weiteren ballistischen Untersuchungen die Beanspruchung durch Splittereinschlag, mit der bei einer Explosion von Sprengkörpern im Transportbehälter zu rechnen ist, betrachtet. Diese tritt gegenüber dem Gasdruck nur lokal auf. Die punktuellen Belastungen müssen durch die Behälterwand, also das entsprechende Textil, ebenfalls vorwiegend in Verformungsarbeit umgewandelt werden, ohne dass hierbei das Textil selbst zerstört wird.

Alle Ergebnisse dieser drei Belastungsversuche dienten der Herleitung eines textilen Kombinationsmaterials. Aufgrund der Anforderungen und Testergebnisse insgesamt haben sich hierbei Aramid-Fasergarne und die daraus hergestellten textilen Flächen als besonders geeignet herausgestellt. Für die textilen Flächengebilde konnte so die vorzugsweise Verwendung von direkt orientierten multiaxialen Gelege (vgl. Abb. 3) und biaxialen Gewebestrukturen (vgl. Abb. 4) herausgestellt werden. 


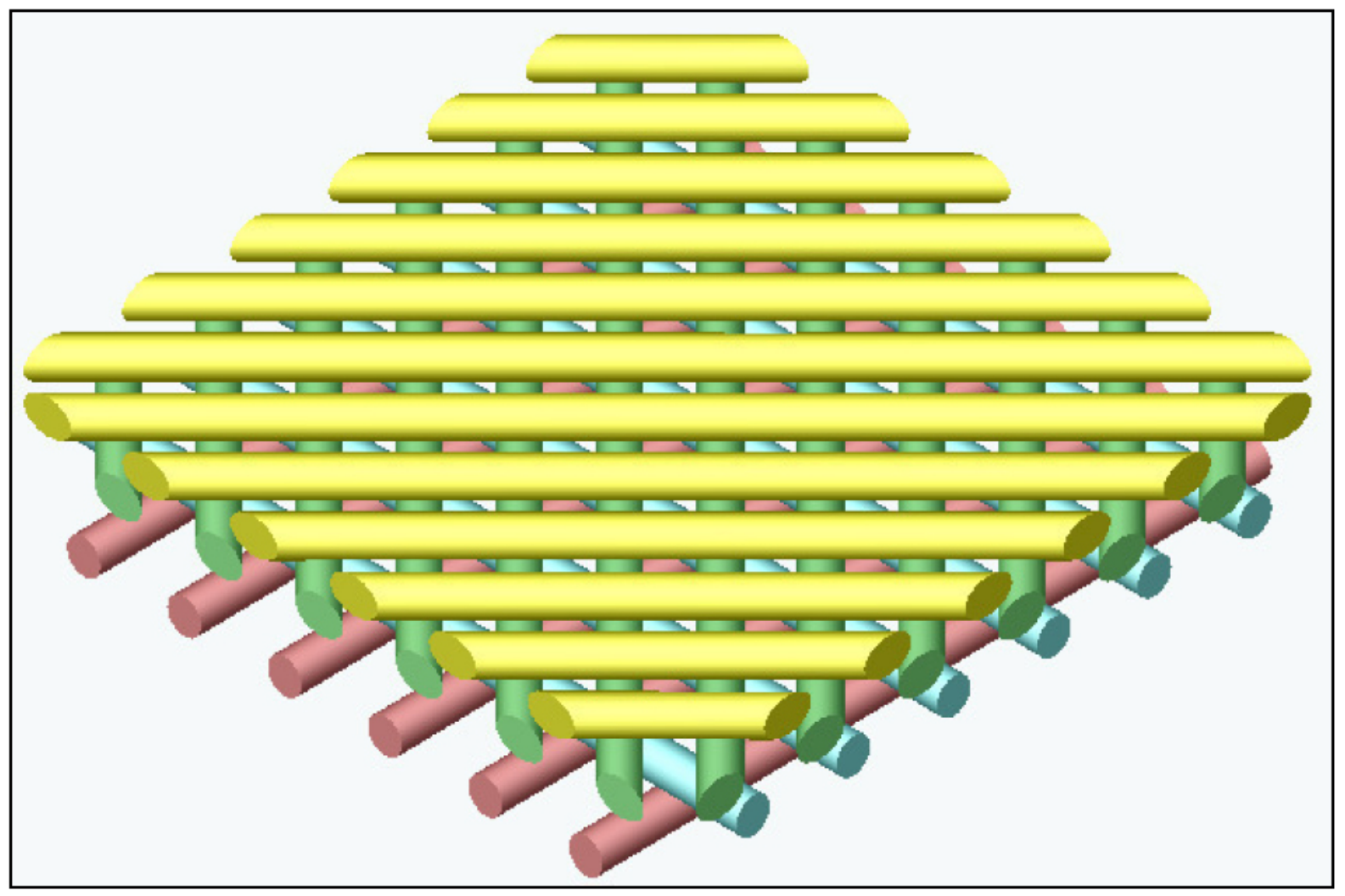

Abbildung 3: Entwurf einer multiaxialen Flächestruktur

Aufbauend auf diesen Erkenntnissen konnten unter Verwendung der Ergebnisse der Betrachtungen zu den logistischen Anforderungen und Bedingungen umfangreiche Untersuchungen, Musterfertigungen und kurzzeitdynamische Belastungstests zu explosionsfesten Verschlusssystemen für mögliche textile Transportbehälter stattfinden. Unter Verwendung von Aramid-Gewebe wurden systematisch erarbeitete Lösungen umgesetzt und im Berstversuch getestet. Im Ergebnis dieser Betrachtungen ergaben sich schlitzförmige Behälteröffnungen, die durch beidseitig angebrachte Druckplatten mittels Verschrauben derselben geschlossen werden, als außerordentlich zweckmäßig.

Da sich das Aramid-Gewebe als besonders geeignetes Material herausgestellt hat, konnten durch Einsatz der KEMAFIL-Technik spezielle Aramid-Garnstrukturen angefertigt werden, die als Ausgangsmaterialien für kombinierte Flächengebilde dienten. Durch die KEMAFIL-Technik lassen sich hohe Materialdichten in den Garnstrukturen erzeugen, welche für die Weiterverarbeitung zu kurzzeitdynamisch hochbelastbaren, sprich explosionssicheren, textilen Flächengebilden benötigt werden. Unter Verwendung der Multiaxialtechnik wurden die Aramid-KEMAFIL-Garne zu triaxialen Gelegen ausgebreitet und in Verbindung mit der Nähwirktechnik zu einer textilen Fläche verarbeitet. Speziell die Herstellung der textilen Flächengebilde nach der Nähwirktechnologie gestattet durch die vielfältigen Mustermöglichkeiten bei der Verlegung direkt orientierter biaxialer und multiaxialer Fadensysteme eine hohe Variabilität der Flächenstrukturen und somit die Konstruktion von spezifischen Materialkombinationen. 


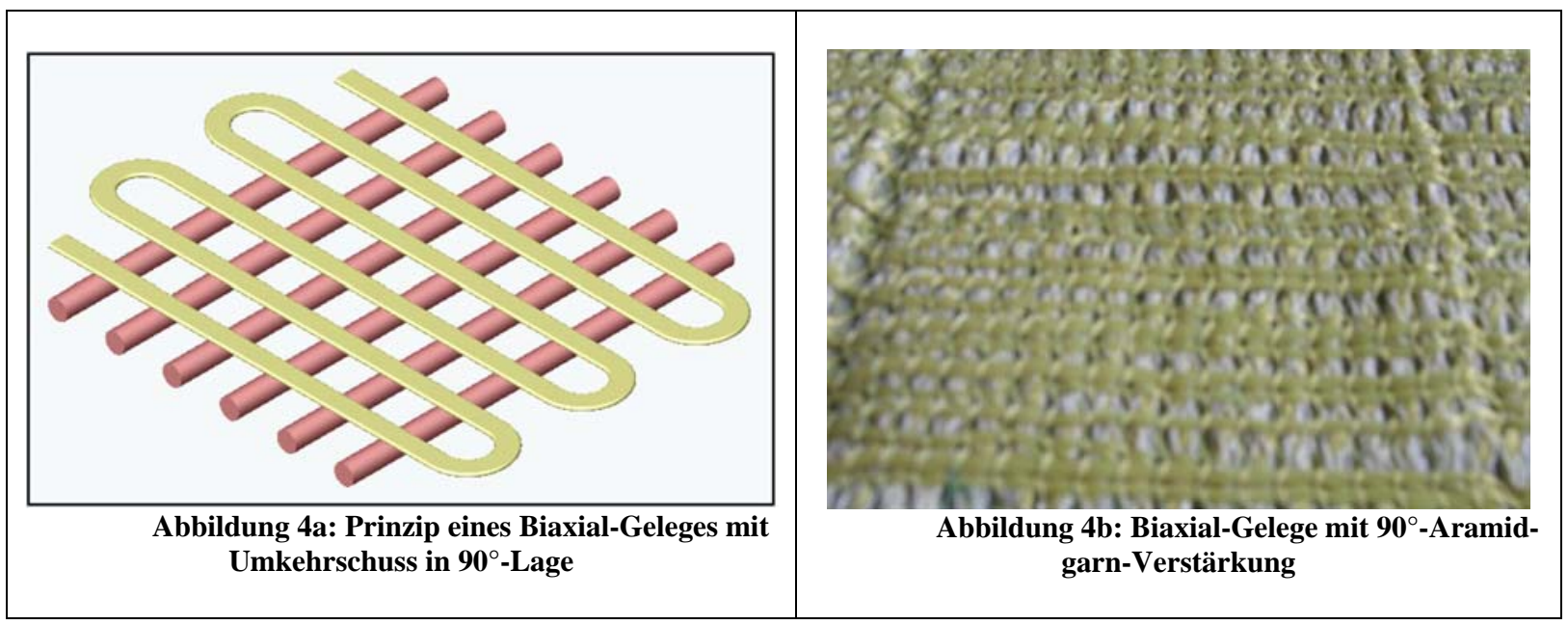

Im Ergebnis der umfangreichen Arbeiten zu den einzelnen textilen Konstruktionen konnten nach einem ersten kalkulatorischen Ansatz spezielle Kombinationsmaterialien in ihrer Gestaltung beschrieben werden.

Aus der Zusammensetzung mit Aramidvliesen und Aramidgeweben sind textile Konstruktionen entstanden, die zur Herstellung von zwei Test-Musterbehältern verwendet wurden. Dabei bestehen die textilen Konstruktionen in ihrer Makrostruktur aus fünf textilen Lagen oder Schalen, wobei jede einzelne Schale wiederum aus verschiedenen einzelnen Flächengebilden zusammengesetzt ist. In weiterführenden Versuchen sind diese TestMusterbehälter speziellen brandtechnischen Beanspruchungen, einer Belastung durch Splitterbeschuss und einer Belastung durch Explosion einer definierten Menge Sprengstoff (vgl. Abb.5) ausgesetzt worden.

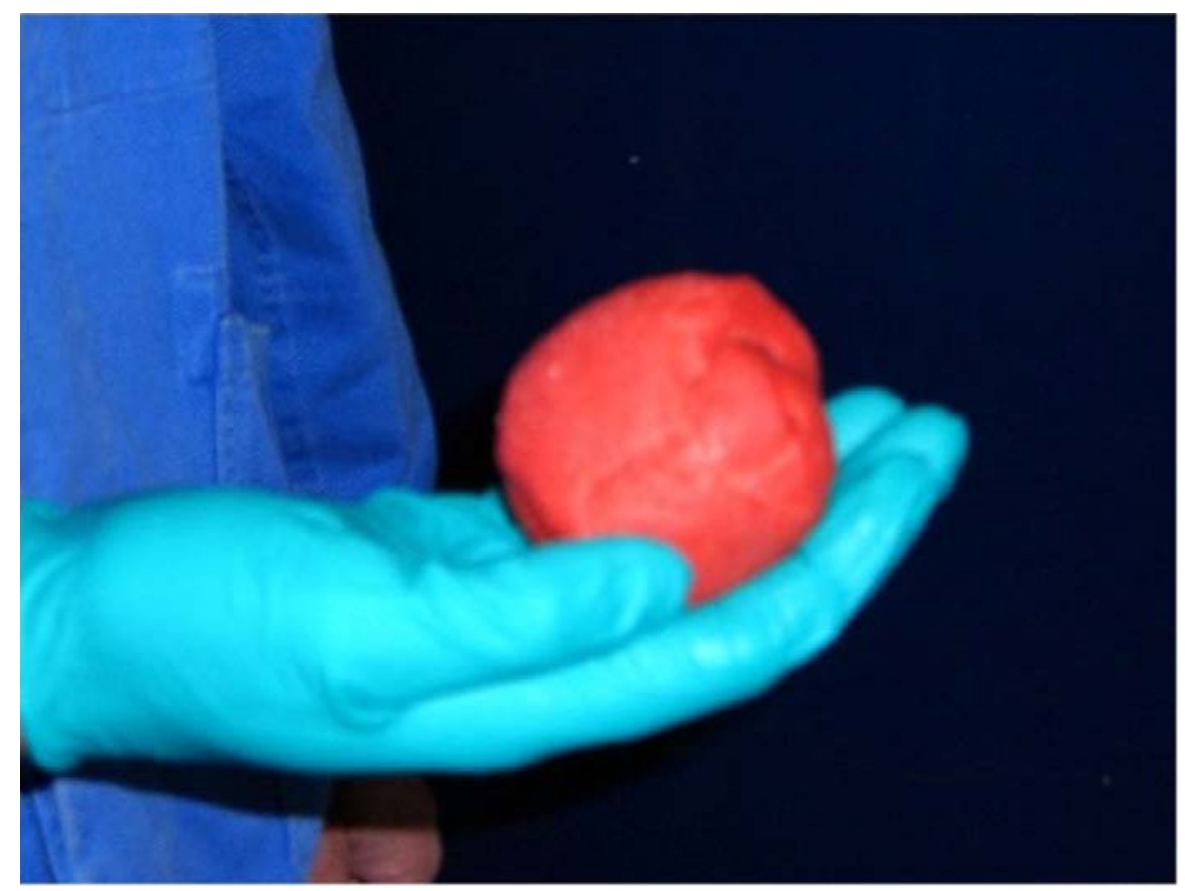

Abbildung 5: Sprengmasse (200g Semtex-Sprengstoff)

Für die brandtechnischen Versuche wurde das textile Kombinationsmaterial als Flächengebilde in einem eigens dazu angefertigten metallischen Testmusterbehälter platziert. Um die äußerst positiven Ergebnisse eines ersten Versuches zu bestätigen, wurde ein zweiter Versuch durchgeführt, bei dem das entwickelte Kombinationsmaterial durch verstärkte Sauerstoffzufuhr lang anhaltend, höheren Temperaturen ausgesetzt wurde. Da verglichen zum ersten Versuch keine höhere Materialschädigung eingetreten ist, konnte von der Haltbarkeit des Materials unter derartigen Hochtemperaturbeanspruchungen ausgegangen werden. 
Im Anschluss wurde das gleiche Material einem Test durch Splitterbeschuss unterzogen. Dabei verwendete Normsplitter dienen der vergleichenden Analyse und der Herleitung von Berechnungsalgorithmen hinsichtlich lokaler, kurzzeitdynamischer Belastungen von Flächengebilden. Den versuchsweise eingetragenen Beanspruchungen hat das entwickelte textile mehrlagige Flächengebilde im vollen Umfang standgehalten.

Aufbauend auf der Summe dieser Erkenntnisse wurde für den Explosionsversuch eine ca. $1,5 \mathrm{~m}^{2}$ große Mantelfläche eines dafür gefertigten, zylinderförmigen Testbehälters vollständig aus dem Kombinationsmaterial zusammengesetzt. In diesem Zusammenhang durchgeführte maschinentechnische Versuche zum Vernähen der einzelnen Schichten zur Mehrlagenkonstruktion mussten abgebrochen werden. Die hierfür vorhandenen technischen Voraussetzungen genügen derzeit nicht, um eine hinreichend stabile Zusammenfassung der einzelnen textilen Flächen zu einem handhabbaren und weiterverarbeitbaren Kombinationsmaterial zu erreichen. Deshalb mussten manuelle Arbeiten zur Fertigstellung der fünfschaligen textilen Ummantelung für den TestMusterbehälter durchgeführt werden. In diesen Behälter wurden 200 g Semtex-Sprengstoff vertikal in der Mitte und horizontal in der Nähe der Behälterwand im Innern des Behälters platziert und zur Detonation gebracht (vgl. Abb. 6). Die damit entstehende komplexe Beanspruchung der textilen Mantelfläche reichte lediglich zur strukturellen Zerstörung der ersten, innersten Schale des Behälters. Alle folgenden vier Schalen der Behälterwand blieben strukturell unbeschädigt. Im Ergebnisvergleich zwischen den brandtechnischen Versuchen unter Einsatz von Hochtemperatur über $2000{ }^{\circ} \mathrm{C}$ und dem Explosionstest konnte somit eine Korrelation hinsichtlich der Belastbarkeit der entwickelten textilen Strukturen festgestellt werden.

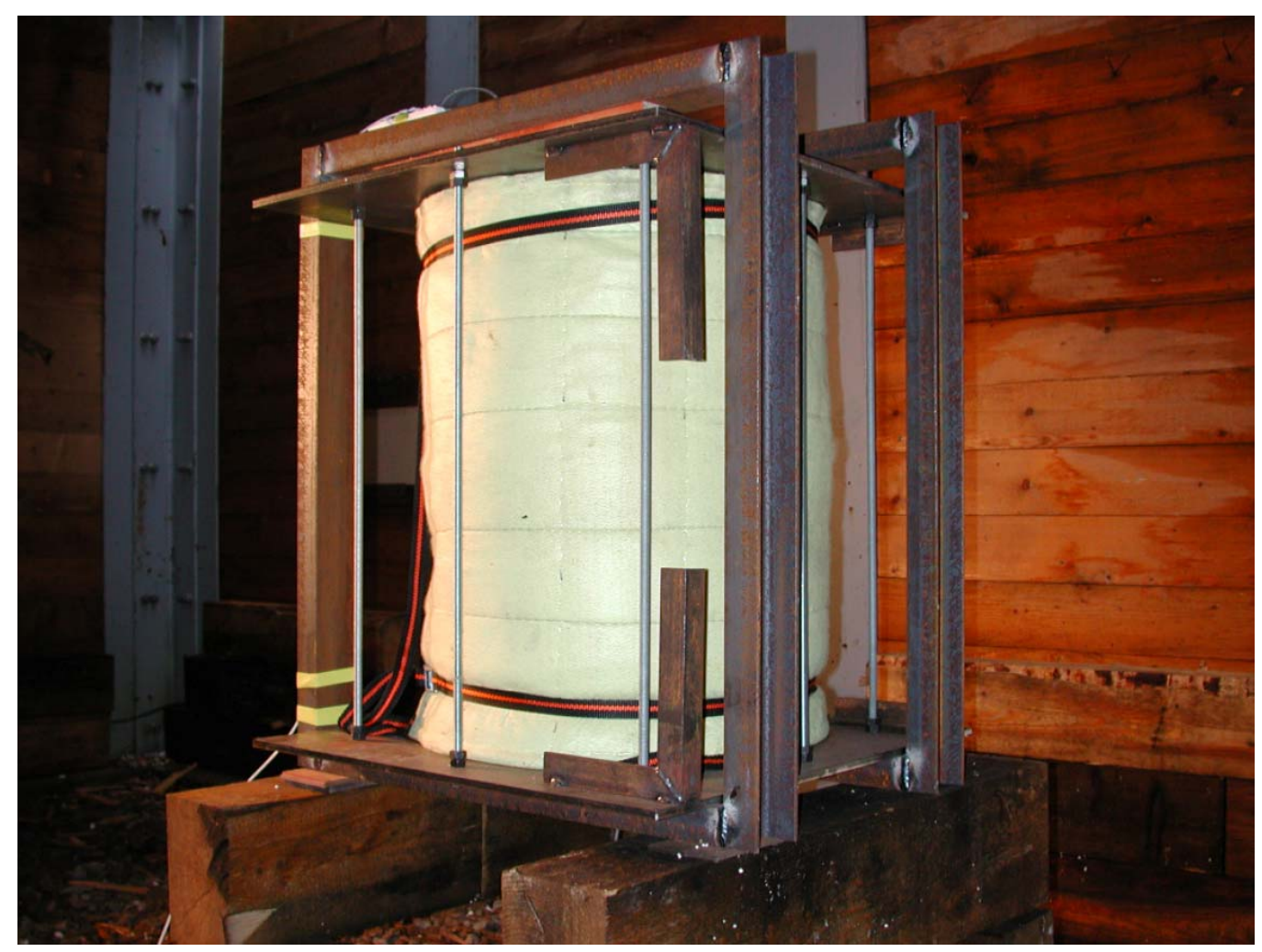

Abbildung 6: Test-Musterbehälter vor dem Versuch mit Stahlrahmen

Hinsichtlich der gewünschten Schutzwirkung sind die speziell entwickelten, textilen Strukturen grundlegend geeignet, um hieraus anwendungsorientierte Entwicklungen, basierend auf den Anforderungen der Distribution und Logistik in Angriff zu nehmen.

Durch die schnelle Umsetzung der Forschungsergebnisse wird die Erschließung neuer Anwendungsfelder für Hersteller von Technischen Textilien und für den Textilmaschinenbau ermöglicht. Ein wichtiges Gebiet ballistischer Schutzsysteme, der Schutz vor Bomben oder Sprengkörpern, welche möglicherweise in Transportmittel eingeschleust und in Gepäckräumen des öffentlichen Personenverkehrs verstaut wurden, kann auf Grundlagen der vorliegenden Ergebnisse mit neuartigen textilen Konstruktionen entwickelt und ausgebaut werden. 
Auf Seiten der Anwendung befinden sich alle Fahrzeuge aus den Bereichen des öffentlichen Luft-, Land- und Wasserverkehrs im Fokus näherer Betrachtungen. Insgesamt lässt sich neben dem Einsatz spezieller Verpackungs- und Transportbehältnisse auch die Auskleidung von Gepäckräumen als mögliche Lösungsvarianten praktischer Umsetzungen ableiten. Die Ergebnisse des Projektes wurden kleinen und mittelständischen Unternehmen der Textil-, Transport- und Verpackungsbranche zur Verfügung gestellt.

\section{Literatur}

[Haag2006]

Haag, J.; Scheer, B.: Der Ruf nach mehr Sicherheit, IN: PackReport 29(2006)4, S. $42-44$ 